\title{
Requerimientos de las Pymes para la formación de contadores profesionales en el contexto actual
}

\author{
Requirements of the Sme's for the formation professional \\ accountants in the current context
}

Roger Manuel Patrón Cortés (roger_patron_cortes@hotmail.com)

Universidad Autónoma de Campeche (México)

Edith J. Cisneros-Cohernour (cchacon@uady.mx)

Universidad Autónoma de Yucatán (México)

http://dx.doi.org/10.12795/EDUCADE.2013.104.07

\begin{abstract}
RESUMEN: Esta investigación tiene como objetivo determinar las competencias que requieren las Pymes del Contador profesional en los tiempos actuales. Este estudio fue exploratorio con enfoque cualitativo y participaron un total de 40 empresas. Se diseñó un cuestionario semi estructurado y una guía de entrevistas con la finalidad de profundizar y encontrar explicaciones a los resultados obtenidos. Se encontró que aunque el plan de estudios del Contador profesional de una universidad ubicada en México, es un programa de calidad que ha sido evaluado en el nivel 1 por los CIEES y Acreditado por el CACECA, las Pymes señalaron que requieren reforzar sus conocimientos en el área fiscal, software y auditoría. Los empresarios consideran que les falta capacidad para administrar y para manejar ciertos programas de cómputo, pues no es lo mismo tener los conocimientos que ponerlos en práctica. Asimismo, indicaron que necesitan reforzar ciertos valores tales como la responsabilidad, la honestidad y la lealtad. Las Pymes sugirieren revisar y actualizar constantemente sus planes y programas de estudio y una mayor vinculación con las empresas.
\end{abstract}

PALABRAS CLAVE: Competencia profesional, Federación Internacional de Contadores, Programas de Educación, Licenciado en Contaduría, Universidad Mexicana.

\begin{abstract}
This study aims to determine the competencies required by SME's to the professional Accountant at the present time. This study was exploratory with qualitative approach and involved a total of 40 companies. We designed a questionnaire semi-structured and an interview guide in order to deepen and to find explanations for the results. We found that although the curriculum of the professional Accountant of a university located in Mexico, is a quality program that has been assessed at level 1 by CIEES and Accredited CACECA, the SMEs said they need reinforce their knowledge on the tax area, software and audit. The employers believe that they lack the capacity to manage and use of software, it is not the same as having the knowledge that implement them. Also they indicated need to reinforce certain values such as responsibility, honesty and loyalty. The SME's suggest constantly review and update their plans and programs of study and more linkages with enterprises.
\end{abstract}

KEYWORDS: Professional Competence, International Federation of Accountants, Education Programs, Bachelor of Accounting, Mexican University

\section{INTRODUCCIÓN}

El mundo globalizado actual, el crecimiento de las empresas multinacionales, la apertura de nuevos mercados, las alianzas estratégicas y la generación de grandes fusiones, entre otros aspectos demandan que las Pequeñas y Medianas Empresas (Pymes) se adapten a los cambios e innovación para poder mantenerse y crecer. 
Esto hace necesario que el Licenciado en Contaduría de las nuevas generaciones cuente con las competencias que les permita ofrecer mayor calidad y mejor capacidad de respuesta a las complejidades de los tiempos modernos. El Licenciado en Contaduría es un profesionista capaz de desarrollar soluciones innovadoras a los problemas contables, de costos, auditoría y fiscales, contribuyendo como agente de cambio estratégico a la dirección efectiva de la empresa y a la maximización del rendimiento del accionista o propietario (Universidad Autónoma de Campeche, 2012).

El Licenciado en Contaduría es un consultor, que participa en las decisiones que definen el rumbo de las empresas, en pocas palabras es un asesor de negocios (Ramos, 2010). La actividad principal del Licenciado en Contaduría se ha caracterizado por ser la de proveer un servicio profesional, sus servicios van enfocados a la generación de información relevante dirigida a diversas personas e instituciones coadyuvando con sus conocimientos y asesoría al desarrollo económico empresarial. Ramos (2010) también señala que el Licenciado en Contaduría tiene que prepararse y desarrollarse en los nuevos ambientes cada vez más competitivos, en el cual conservará y desarrollará su propio campo de acción en la labor de fortalecer a las entidades en su administración, finanzas y control de las operaciones, apoyando con su capacidad profesional y responsabilidad social el crecimiento de las mismas para que puedan hacer frente a los nuevos retos que les depara el futuro. Montero y Zaá (2009: 32) al referirse a la Contaduría Pública señalan que toda profesión no mercantil, basada en la práctica científica, evoluciona hacia formas más refinadas de prestación de servicios y que esto requiere un mayor control de calidad, no sólo para satisfacer la demanda de los clientes, sino para desarrollar de manera óptima, los métodos y procedimientos normativos por los que se rige el ejercicio profesional.

Este trabajo tiene como objetivo determinar las competencias profesionales que requieren las Pymes del Licenciado en Contaduría de una universidad latinoamericana ubicada en México en los tiempos actuales. El desarrollo de este estudio se encuentra estructurado de la siguiente manera: en el segundo epígrafe analizamos la literatura relativa a los requerimientos, las competencias profesionales, los programas de educación y diversas investigaciones sobre el tema en el contexto actual, en el siguiente desarrollamos el diseño metodológico, en el epígrafe cuarto comentamos los resultados obtenidos y finalizamos con las conclusiones al respecto.

La investigación contribuye a la literatura empresarial de las Pymes y a encontrar las competencias profesionales para la formación de los Licenciados en Contaduría de una universidad mexicana, con la finalidad de fortalecer sus programas de estudio en beneficio de un mejor desempeño en su actividad profesional que coadyuve al desarrollo económico y sustentable de su Estado y de México, pues como señala Quirós (2006) cada programa de estudio tiene la responsabilidad de proponer una perspectiva de formación integral del Contador Público.

\section{REVISIÓN DE LA LITERATURA}

\subsection{ReQUeRIMIENTOS PROFESIONALES DE LAS PYMES DEL LICENCIADO EN CONTADURÍA}

El Licenciado en Contaduría puede ejercer en el sector público y privado, por tanto su preparación debe ser integral para desempeñarse con éxito en todas las organizaciones. Antognolli (2008) señala que la profesión del Licenciado en Contaduría los prepara fundamentalmente como asesores legales en materia comercial, laboral y especialmente impositiva, además los forma para exponer informes ante organismos oficiales y realizar tareas de auditoría, entre otras actividades. No obstante, en el contexto económico actual la necesidad de innovar, generar riqueza y desarrollar el potencial creativo se asocia con mayor fuerza a las Pymes, las cuales deben estar provistas de profesionales con una alta capacidad innovadora y emprendedora para sobrevivir y crecer.

educade, no 4, 2013, p. 98 
Hernández (2009) indica que las Pymes necesitan administrar eficientemente sus obligaciones fiscales como parte de su gestión competitiva, por tanto los Licenciados en Contaduría requieren conocer la legislación tributaria, normas y procedimientos; y operar eficazmente los programas informáticos para hacer más efectiva su labor profesional. Los Contadores Públicos deben facilitar la formalización de las empresas y el logro de la disciplina tributaria. Con el apoyo de los Licenciados en Contaduría, las Pymes deben registrar adecuadamente sus operaciones y hacer transparente su manejo financiero de tal forma que pueden ser auditadas fácilmente por todos los interesados de la empresa, evitando multas y usando los beneficios económicos definidos para los regímenes establecidos, como por ejemplo poder recibir la devolución de ciertos impuestos, entre otros aspectos. Asimismo, los licenciados en contaduría deben contribuir para que las empresas cumplan en tiempo y forma con sus declaraciones de impuestos, permitiendo mediante su buena imagen obtener créditos. Además el licenciado en contaduría requiere contar con un adecuado manejo administrativo, laboral y contable para hacer competitiva a su empresa con proyección de crecimiento.

Según Lopera, Granda, Castaño y Rodríguez, (2009: 42) en la formación actual del Licenciado en Contaduría se hace énfasis en la necesidad de representar la realidad empresarial con modelos y metodologías contables, matemáticas, financieras, fiscales, administrativas, sociales y económicas, todas éstas con apoyo a la toma de decisiones para así brindar información de una manera óptima, clara y precisa. Por otra parte, el proceso de mundialización de los mercados y los avances tecnológicos han generado cambios en los requerimientos para la formación de los Licenciados en Contaduría. La internacionalización de las organizaciones está demandando de las Instituciones de Educación Superior (IES) nuevos enfoques en sus programas curriculares.

Biedma, Gómez y Ruiz (2011: 113) indican que se ha destacado la necesidad de que la formación universitaria haga un mayor esfuerzo en proporcionar al estudiante herramientas y recursos que lo hagan más capaz de desarrollar con éxito un trabajo profesional acorde a su perfil y que con esta finalidad los profesores universitarios deberán asumir el reto de formar a los futuros profesionales basados en competencias, mediante un conjunto de conocimientos, habilidades y actitudes que deberá ser trasvasado y desarrollado por el estudiante durante su vida académica.

De acuerdo con De Agüero (2001: 33) las instituciones de educación superior están enfrentando retos que las han obligado a reflexionar sobre la necesidad de hacer cambios en los procesos de enseñanza aprendizaje para adaptarlos a las nuevas condiciones del mercado. Asimismo, a través de la Organización Mundial del Comercio (OMC) se están exigiendo nuevas prácticas en los servicios profesionales y la aplicación de normas de conducta que aseguren el cumplimiento de los estándares mundiales de calidad de estos servicios.

Las Pymes demandan de sus futuros Licenciados en Contaduría conocimientos, habilidades y valores que en ocasiones están muy alejados de lo que aprenden en la escuela. Ibarrondo (2011: 35) señala que la interrelación entre la teoría y la práctica profesional en el ámbito de la Contabilidad resulta necesaria y conveniente. Debido a que se tienen pocos estudios sobre el desempeño de estos profesionistas, se decidió investigar si cubren los requerimientos en su desempeño profesional en las Pymes.

\subsection{COMPETENCIAS PROFESIONALES}

La Organización para la Cooperación y el Desarrollo Económico (OCDE, 2005) reconoce la complejidad de las condiciones de vida actuales, lo que llevó al planteamiento del concepto de competencia, que involucra la habilidad de enfrentar demandas complejas apoyándose y movilizando recursos psicosociales que incluye destrezas y actitudes en un contexto particular. Al manejo de herramientas, tanto físicas como socioculturales, se agrega su comprensión y adaptación a los propios

educade, no 4, 2013, p. 99 
fines de las personas y a su uso interactivo. Además de interactuar con grupos heterogéneos, las personas necesitan tomar la responsabilidad de manejar sus propias vidas, situarlas en un contexto social más amplio y conducirse de manera autónoma, se espera que las personas se adapten a los continuos contextos variables y que den muestras de creatividad e innovación, así como de automotivación y valores, superando la sola reproducción del conocimiento acumulado.

De acuerdo con Ghersi y Aguirre (2010) el concepto de "competencia" en los currículos de carreras del espacio Europeo de Educación Superior pareciera estar aplicándose ya por más de una década, con distintos niveles de alcance y de satisfacción en la evaluación de los resultados de aprendizajes y no se vislumbra posibilidad de retorno.

En años recientes muchas escuelas han modificado sus modelos educativos y un gran número de IES han migrado a modelos de educación basados en competencias. Allende y Morones (2006: 4) definen las competencias como el "conjunto de conocimientos, habilidades y destrezas, tanto específicas como transversales, que debe reunir un titulado para satisfacer plenamente las exigencias sociales". Fomentar las competencias es el objetivo de los programas educativos. Las competencias son capacidades que la persona desarrolla en forma gradual y a lo largo de todo el proceso educativo y son evaluadas en diferentes etapas. Pueden estar divididas en competencias relacionadas con la formación profesional en general (competencias genéricas) o con un área de conocimiento (específicas de un campo de estudio).

Según Tobón, Pimienta y García (2010) actualmente las competencias se entienden como actuaciones integrales para identificar, interpretar, argumentar y resolver problemas del contexto con idoneidad y ética, integrando: a) el saber conocer, que es la puesta en acción, a través de la actuación de un conjunto de herramientas necesarias para procesar la información de manera significativa acorde con las expectativas individuales, las propias capacidades y los requerimientos de una situación en particular, se diferencia de los conocimientos específicos y de la memorización de la información y se divide en procesos cognitivos, instrumentos cognitivos y estrategias cognitivas y metacogntivas, b) el saber hacer, que consiste en saber actuar con respecto a la realización de una actividad o resolución de problemas, comprendiendo el contexto y teniendo como base la planeación, asimismo se caracteriza por basarse en la toma de conciencia y el control a través de la continua planeación, monitoreo y evaluación de lo que se hace, también se divide en procesos de desempeño, instrumentos de actuación y estrategias, y c) el saber ser, que consiste en la articulación de diversos contenidos afectivos-motivacionales, enmarcados en el desempeño competencial y se caracteriza por la construcción de la identidad personal, la conciencia y control del proceso emocional-actitudinal en la realización de una actividad.

Estudios realizados por la International Federation of Accountants (IFAC) (1998: 17) consideran que la educación y experiencia del Contador Público se configura a partir de conocimientos, habilidades y valores profesionales. En cuanto a lo cognitivo se plantea la formación en conocimiento general, organizacional y de negocios, tecnologías de la información y la contabilidad con sus áreas relacionadas. Se espera que el Contador Público tenga habilidades intelectuales, interpersonales y de comunicación. Asimismo, debe contar con un comportamiento ético, valores profesionales y experiencia. Se tiene como objetivo la competencia profesional del Contador capaz de hacer una contribución positiva a la profesión y a la sociedad. Es necesario que el Contador Público desarrolle sus competencias para identificar problemas y buscar soluciones, apoyado en el diseño y estructuración de información y en su análisis e interpretación para lograr sus objetivos. La orientación profesional se centra en el desarrollo de habilidades y destrezas tendientes a que los estudiantes sean conscientes de sus responsabilidades, conocimientos y valores exigidos para crear juicios y solucionar problemas con integridad. Los estándares internacionales de 
educación para Contadores profesionales pretenden definir las prácticas generalmente aceptadas en la educación para la formación en competencias. Establecen los elementos esenciales que garanticen el reconocimiento y la aceptación internacional del ejercicio profesional que si bien no son de cumplimiento obligatorio, constituyen una referencia autorizada para los reguladores locales.

\subsection{LA FACULTAD DE CONTADURÍA Y ADMINISTRACIÓN DE UNA UNIVERSIDAD MEXICANA Y SU PROGRAMA DE ESTUDIO BASADO EN COMPETENCIAS}

La Facultad de Contaduría y Administración de una universidad ubicada en México tiene sus orígenes desde el año de 1965, tomando como base en sus inicios los programas de estudio de la Universidad Nacional Autónoma de México (UNAM). Con el paso del tiempo ha tenido cambios en los mismos pasando de planes anuales a semestrales, en 1988 se convirtió en Facultad ofertando diplomados y maestrías. También se han realizado diversos estudios socio económicos en la región con el apoyo de académicos, empresarios y dependencias gubernamentales logrando entre otros cambios la actualización de planes de estudios de la carrera de Contador Público versión 2000, la apertura de la Licenciatura en Administración y Finanzas, la apertura de la Maestría en Administración, y los planes flexibles 2006 (Universidad Autónoma de Campeche, 2011).

La capacidad emprendedora es una de las 15 competencias genéricas del perfil universitario para el nivel de licenciatura que la universidad mexicana ha definido. Asimismo, de las 13 competencias profesionales definidas para la Licenciatura en Contaduría, seis se refieren al área financiera, tres al área contable, dos al área de costos, una al área fiscal y la última señala profundizar y facilitar los procesos de investigación, adopción e innovación tecnológica para incrementar la productividad y competitividad de las empresas. El plan de estudios vigente 2009 de la Licenciatura en Contaduría, es flexible, por competencias, centrado en el aprendizaje y se encuentra estructurado en ocho semestres con un mínimo de 51 unidades de aprendizaje. La carga académica es de 194 créditos incluyendo la práctica profesional. Las unidades de aprendizaje se encuentran estructuradas de forma secuencial con la finalidad de que los estudiantes generen su propia síntesis de lo aprendido. En las etapas avanzadas del programa, los estudiantes pueden escoger para su formación un poco más especializada, materias ofertadas dentro de los bloques de las optativas de Auditoría y Fiscal, Costos y Contabilidad y Finanzas. Extracurricularmente el alumno debe acreditar las competencias en Microsoft: Word, Excel y Power Point. Así como también cumplir con el servicio social de acuerdo con la legislación universitaria. Este programa educativo de calidad ha sido evaluado en el nivel 1 por los Comités Interinstitucionales para la Evaluación de la Educación Superior, A. C. (CIEES) y Acreditado por el Consejo de Acreditación de la Enseñanza en la Contaduría y Administración, A.C. (CACECA) (Universidad Autónoma de Campeche, 2012).

\subsection{INVESTIGACIONES SOBRE REQUERIMIENTOS DEL CONTADOR PROFESIONAL}

Una investigación realizada por Quirós (2006) relativa a las competencias formativas y el mercado profesional contable reveló que la formulación y desarrollo de competencias es un problema no solo técnico, sino que moviliza concepciones pedagógicas. Los estándares proponen unos requisitos mínimos de calidad, pero el diseño de competencias debe ser un proceso más integral que recoja las distintas dimensiones de formación. Con la normatividad en materia de estándares se abre un debate de las implicaciones pedagógicas, económicas y sociales que tiene la formación del Contador Público. Por tanto cada programa de Contaduría Pública tiene que hacer un balance de dichas circunstancias, tomar decisiones y asumir las consecuencias requeridas. Los procesos de formulación de competencias comprometen a diferentes sectores de la sociedad tales como la empresa, la 
comunidad académica y el Estado, entre otros. La universidad tiene la responsabilidad de captar esas expectativas y proponer una perspectiva de formación integral del Contador Público.

Un estudio desarrollado por Bernabeu (2009) tuvo como objetivo indagar sobre la opinión de dirigentes de empresas industriales, comerciales y de servicios, así como también de funcionarios gubernamentales y egresados recientes, respecto de las competencias laborales que son requeridas actualmente a los Contadores Públicos ya que según su opinión difieren de los diseños curriculares de la carrera. Encontró que las nueve competencias más demandadas por orden de importancia fueron: a) solidez en los conocimientos y habilidades contables y administrativas, b) adaptabilidad al cambio, c) compromiso, d) ética, e) calidad del trabajo, f) idoneidad en los conocimientos y habilidades legales, financieras y económicas, g) comunicación, h) aprendizaje continuo, e i) uso experto de software contable e impositivo. Los diferentes grupos que opinaron ponen en evidencia preferencias dispares en la comparación inter-grupos, en cambio observó cierta homogeneidad al interior de cada grupo. Esto se explica debido al amplio espectro de posibilidades que tiene el campo laboral del Contador Público, lo cual genera objetivos bien diferenciados según de qué tarea se trate, por tanto, quienes contratan los servicios de un Contador Público demandan distintas competencias prioritarias.

Otro trabajo elaborado por Valera (2009) basado en el análisis de macrotendencias propone las siguientes competencias profesionales para un egresado de la carrera de Contabilidad y Finanzas: a) comunicativa: facilidades de comunicación oral y escrita, trabajo en equipo, liderazgo, capacidad de desarrollar relaciones públicas y suministrar datos de calidad oportunos, comprensibles y transparentes, b) cognitiva: gestión de proyectos, iniciativa, creatividad, adaptación al cambio, independencia, y formación jurídica, c) indagativa: dominio de tecnologías de información, análisis financiero, diseño de sistemas contables y de control interno, manejo de la documentación primaria, pertinencia, pertenencia, y ofrecer diagnósticos sobre riesgos y rentabilidad de las empresas, d) argumentativa: negociador, facilitador de la toma de decisiones, explicar situaciones contables-financieras utilizando lenguaje de negocios, e interpretar situaciones contable-financieras medioambientales y sociales y actuar en consecuencia e) propositiva: identificar situaciones problemáticas de su profesión, formular un banco de problemas inherentes a su profesión atendiendo las características de su entorno laboral, conjugación del desarrollo teórico con capacidades, habilidades y recursos para atender las necesidades de la sociedad, resolución de problemas dados en su ámbito profesional utilizando avances científicostécnicos de su ciencia, y asumir responsabilidad frente a las soluciones propuestas, y f) investigativa: uso de métodos de investigación científica en su esfera de actuación, participación en eventos científicos y cursar estudios de posgrado. Resalta la necesidad de perfeccionar la profesión utilizando la investigación científica

Un trabajo hecho por Ghersi y Aguirre (2010) cuyo propósito fue determinar si el egresado de la carrera de Contador Público posee las competencias necesarias para desenvolverse en su ámbito laboral, encontró que si bien los egresados no tienen las habilidades de tipo práctico necesarias, es decir tienen pocas competencias en ese sentido; reconocen en su formación, valiosos elementos relativos a su flexibilidad profesional y aptitud para la resolución de situaciones concretas. Los autores afirman que sus propias cualidades en la mayoría de los casos, y en segundo lugar los conocimientos adquiridos han sido determinantes a la hora de su inserción profesional. Diferentes grupos refirieron que dichas capacidades personales se nutrieron e incrementaron en la relación con pares, profesores y conocimientos, hasta lograr las competencias adecuadas. 


\section{METODOLOGÍA}

Los tiempos actuales están demandando servicios profesionales de calidad, acordes con una sociedad cambiante caracterizada principalmente por la globalización y regionalización. Por tanto, los profesionistas deben incorporar aspectos tales como la acreditación de planes y programas de estudio, la actualización, la certificación y la vinculación, entre otros. (Fernández, 2001:35). A pesar de que se realizaron algunos estudios regionales en la universidad mexicana que sirvieron de base para adaptar los planes de estudio del año 2000 y abrir la Licenciatura en Administración y Finanzas, entre otras acciones, mayor investigación es necesaria debido a que han pasado más de diez años y los requerimientos de los tiempos actuales son diferentes. Por tanto, se decidió realizar este estudio que tiene como objetivo determinar las competencias que requieren las Pymes del Licenciado en Contaduría de una universidad ubicada en México en los tiempos actuales.

Este estudio es exploratorio con enfoque cualitativo y se realizó en el mes de enero del año 2012 con la participación de 40 directores generales de empresas pequeñas y medianas, de las cuales 22 (54\%) son de servicios, 13 (33\%) son comerciales y 5 (13\%) son productivas.

Se diseñó un cuestionario con preguntas semi estructuradas y se elaboró un análisis de la información documental. El instrumento inicial fue sometido a una prueba piloto permitiendo eliminar las preguntas poco claras o ambiguas y mejorar su planteamiento. Asimismo, se elaboró una guía de entrevistas con la finalidad de profundizar y encontrar explicaciones a los resultados obtenidos. El estudio cualitativo permitió "reforzar teorías e identificar cuestiones críticas para investigaciones futuras" (Stake, 1994: 245).

Para la aplicación de los cuestionarios, se solicitó la ayuda de los alumnos de la universidad mexicana. Los cuestionarios se administraron en las empresas previa cita, además se realizaron entrevistas personalizadas con los sujetos de estudio. La información se organizó mediante categorías y tablas de frecuencia permitiendo identificar y analizar los objetivos de la investigación.

\section{RESULTADOS}

En cuanto a si existe preferencia en la contratación del Licenciado en Contaduría (LC) por alguna institución de procedencia, 19 empresas (47\%) señalan que no tienen preferencias, pues lo que les interesa es que tengan los conocimientos, la experiencia y los valores éticos adecuados, mientras que 21 empresas (53\%) tienen preferencia por los egresados de la universidad mexicana debido a su nivel de preparación y desempeño laboral. Por otra parte, 27 empresas (68\%) señalan que los LC tienen los conocimientos que exigen los tiempos actuales para que sus empresas sean competitivas, mientras que 13 empresas $(32 \%)$ comentan que requieren adquirir otros conocimientos. Los conocimientos que requieren adquirir para poder ser contratados por las empresas se observan en la Tabla 1.

TABLA 1CONOCIMIENTOS QUE REQUIEREN ADQUIRIR LOS LC.

\begin{tabular}{lll}
\hline Área & No. & $\%$ \\
\hline Fiscal & 22 & 55 \\
Software & 9 & 23 \\
Auditoría & 4 & 10 \\
Inglés & 3 & 7 \\
Finanzas & 2 & 5 \\
\cline { 2 - 3 } Total & 40 & 100 \\
\hline
\end{tabular}

Fuente: Elaborado a partir de datos recolectados por los investigadores. 
En la Tabla 1 se aprecia que los LC requieren mayores conocimientos en fiscal, software y auditoría. Los requerimientos de inglés y finanzas se señalan poco. Al respecto se tienen los siguientes testimonios:

"Aunque se supone que los Licenciados en Contaduría son los expertos en estos temas mayor especialización es necesaria".

"Muchas actividades empresariales están manejándose a través de sistemas de cómputo, por tanto es necesario adquirir los conocimientos para operarlos"

"Estos conocimientos son indispensables para el desempeño de la contaduría pública y me parece que se requiere una mayor especialización en ellos".

Los empresarios consideran que 18 (45\%) de los LC tienen las habilidades profesionales que exigen los tiempos actuales para que sus empresas sean competitivas, mientras que 22 (55\%) opinan que requieren adquirir ciertas habilidades. Las habilidades que requieren adquirir se detallan en la Tabla 2.

TABLA 2. HABILIDADES QUE REQUIEREN ADQUIRIR LOS LC

\begin{tabular}{lcc}
\hline Competencia & No. & $\%$ \\
\hline Análisis e interpretación de información & 12 & 30 \\
Relaciones públicas & 12 & 30 \\
Numérica & 8 & 20 \\
Comunicación oral y escrita & 6 & 15 \\
Manejo de software & 2 & 5 \\
\cline { 2 - 3 } Total & 40 & 100 \\
\hline
\end{tabular}

Fuente: Elaborado a partir de datos recolectados en la investigación.

En la Tabla 2 se percibe que las habilidades que requieren adquirir los LC son el análisis e interpretación de la información, relaciones públicas y habilidad numérica entre otras. Como prueba se tienen las siguientes evidencias:

"La interpretación de la información contable es muy valiosa para la toma de decisiones, pero muchos profesionales de la Contaduría sólo elaboran los estados financieros para cumplir con los requisitos de forma y el pago de impuestos."

"El profesional de la Contaduría que logra establecer excelentes relaciones públicas tiene asegurado el éxito de su vida profesional".

"La habilidad numérica es requisito indispensable del Contador, pues es la base de su formación profesional".

Las Pymes manifiestan que $35(87 \%)$ de los LC tienen los valores requeridos por los tiempos actuales, mientras que $5(13 \%)$ comentan que requieren reforzar ciertos valores. Los valores que necesitan reforzar se especifican en la Tabla 3.

TABLA 3. VALORES QUE REQUIEREN REFORZAR LOS LC

\begin{tabular}{lcc}
\hline Valor & No. & $\%$ \\
\hline Responsabilidad & 14 & 35 \\
Honestidad & 12 & 30 \\
Lealtad & 7 & 17 \\
Tolerancia & 4 & 10 \\
Respeto & 3 & 8 \\
Total & 40 & 100 \\
\hline
\end{tabular}

Fuente: Elaborado a partir de datos recolectados en la investigación.

En la Tabla 3 se nota que los valores más requeridos por los empresarios son la responsabilidad, la honestidad, y la lealtad, por citar algunos. Cómo ejemplo se tienen los siguientes argumentos: 
"Ciertos despachos contables realizan los pagos de impuestos de diversas empresas, pero resulta que en ocasiones cuando se les da el dinero no los hacen a tiempo".

"No todos los profesionistas son serios y responsables y sobre todo puntuales en el horario de entrada".

"Si los Licenciados en Contaduría tienen valores aman a la empresa, les gusta su trabajo, lo hacen bien y lo disfrutan, asimismo no sienten que la sociedad los está explotando".

Los empresarios consideran que $28(70 \%)$ de los LC tienen las competencias profesionales que exigen los tiempos actuales para que sus empresas sean competitivas, mientras que 12 (30\%) opinan que requieren adquirir otras competencias. Las competencias que requieren adquirir se detallan en la Tabla 4.

TABLA 4. COMPETENCIAS QUE REQUIEREN ADQUIRIR LOS LC

\begin{tabular}{lll}
\hline Competencia & No. & $\%$ \\
\hline Administrar & 13 & 32 \\
Manejo de software & 9 & 22 \\
Financiamiento & 4 & 10 \\
Investigación & 4 & 10 \\
Impuestos & 4 & 10 \\
Relaciones públicas & 3 & 8 \\
Auditoría & 3 & 8 \\
Total & 40 & 100 \\
\hline
\end{tabular}

Fuente: Elaborado a partir de datos recolectados en la investigación.

En la Tabla 4 se percibe que las competencias que requieren adquirir los LC son la capacidad para administrar y el manejo de software entre otros. Como prueba se tienen las siguientes evidencias:

"Una cosa son los conocimientos y otra muy distinta como se desempeñan".

"A los Licenciados en Contaduría se les contrata para un determinado trabajo y se espera un buen desempeño, pero la mayoría de las veces les falta experiencia".

"Durante su época de estudios los alumnos no realizan prácticas parecidas a las de la vida real y esto los hace poco competentes o incompetentes"

Las fortalezas y debilidades que tienen los LC en su desempeño y que son percibidas por los empresarios de las Pymes se relacionan en la Tabla 5.

TABLA 5. ForTALEZAS Y DEBILIDADES QUE TIENEN LOS LC

\begin{tabular}{llllll}
\hline Fortaleza & No. & $\%$ & Debilidades & No. & $\%$ \\
\hline Preparación académica & 14 & 35 & Falta de experiencia & 16 & 40 \\
Ética profesional & 12 & 30 & Falta de actualización & 12 & 30 \\
Eficiencia & 10 & 25 & Falta de capacitación & 8 & 20 \\
Razonamiento & 4 & 10 & Falta de valores & 4 & 10 \\
\cline { 2 - 4 } & 40 & 100 & Total & 40 & 100 \\
\hline
\end{tabular}

Fuente: Elaborado a partir de datos recolectados en la investigación.

En la Tabla 5 se distingue que la preparación académica de los LC es su principal fortaleza seguida de su ética profesional, eficiencia y razonamiento. Mientras que la falta de experiencia, actualización, capacitación y valores son sus principales debilidades. Las siguientes declaraciones son un ejemplo:

"Generalmente los Licenciados en Contaduría no suelen quedarse en la empresa donde se les brindó la oportunidad de obtener experiencia". 
"La ciencia avanza y diario surgen nuevos conocimientos, nuevas técnicas, nuevos sistemas, lo único constante es el cambio".

"Muchos profesionistas de la contaduría tienen la idea de que se les pondrá un trabajo fácil donde no tendrán que hacer gran cosa pero cuando ven la magnitud del mismo no regresan".

Las oportunidades y amenazas que tienen los LC en su desempeño y que son identificadas por los empresarios se muestran en la Tabla 5.

TABLA 6. OPORTUNIDADES y AMENAZAS QUe TIENEN LOS LC

\begin{tabular}{llllll}
\hline Oportunidades & No. & $\%$ & Amenazas & No. & $\%$ \\
\hline Mejorar su desempeño & 15 & 38 & Competencia & 18 & 45 \\
Actualizarse & 12 & 30 & Avance tecnológico & 10 & 25 \\
Mejorar sus conocimientos & 10 & 25 & Corrupción & 6 & 15 \\
Reforzar sus valores & 3 & 7 & Desempleo & 6 & 15 \\
\cline { 2 - 4 } & 40 & 100 & Total & 40 & 100 \\
\hline
\end{tabular}

Fuente: Elaborado a partir de datos recolectados en la investigación.

En la Tabla 6 se advierte que de acuerdo a la opinión de los empresarios, los LC tienen oportunidad de mejorar su desempeño, actualizarse, mejorar sus conocimientos y reforzar sus valores. Mientras que la competencia, el avance tecnológico, la corrupción y el desempleo son sus principales amenazas. Al respecto los empresarios expresaron:

"Cada día hay más Licenciados en Contaduría egresados de otras escuelas con nuevos y mejores conocimientos"

"A los Licenciados en Contaduría se les da confianza pues manejan recursos de la empresa y puede prestarse a fraudes y actos ilícitos".

"Conozco Licenciados en Contaduría que llevan hasta un año de haber egresado y aún no consiguen empleo, otros trabajan en áreas que no tienen nada que ver con su carrera universitaria"

En las entrevistas cualitativas, se encontró que 5 (13\%) de los gerentes no se encuentran del todo satisfechos con el desempeño que tienen los LC que trabajan en sus empresas por la falta de eficiencia, conocimientos y actitud. Mientras que 35 (87\%) se encuentran satisfechas porque son profesionistas responsables, tienen los conocimientos necesarios, son confiables y por el prestigio de su escuela.

Las recomendaciones de las Pymes para mejorar la formación de los LC se presentan en la Tabla 7.

TABLA 7. RECOMENDACIONES PARA MEJORAR LA FORMACIÓN DE LOS LC

\begin{tabular}{lll}
\hline Recomendaciones & No. & $\%$ \\
\hline Actualización & 16 & 40 \\
Vinculación & 14 & 35 \\
Práctica & 6 & 15 \\
Capacitación & 4 & 10 \\
\cline { 2 - 3 } Total & 40 & 100 \\
\hline
\end{tabular}

Fuente: Elaborado a partir de datos recolectados en la investigación.

En la Tabla 7 se observa que las recomendaciones principales de las Pymes para mejorar la formación de los Licenciados en Contaduría son la actualización de planes y programas de estudio, la vinculación empresarial, mayor práctica que complemente los aspectos teóricos y la capacitación continua de los docentes. Los empresarios expresaron los siguientes testimonios: 
"En la escuela se enseñan ciertos conocimientos pero cuando egresan los profesionistas ya no aplican por el acelerado cambio del mundo globalizado"

"Es necesario que los planes y programas los relacionen con la práctica profesional a través de talleres, estancias, visitas y certificación de competencias laborales".

"Los profesores deben estar a la vanguardia pues ellos además de transmitir conocimientos y experiencia deben propiciar el desarrollo de las competencias profesionales de los alumnos".

\section{CONCLUSIONES}

Las Pymes no se encuentran interesadas en el prestigio de la institución formadora de los Licenciados en Contaduría, sino que éstos cumplan con las expectativas de un buen desempeño y que contribuyan a alcanzar el éxito de las mismas.

Aunque en el caso específico del programa de estudios del Licenciado en Contaduría de una universidad ubicada en México es un programa educativo de calidad que ha sido evaluado en el nivel 1 por los Comités Interinstitucionales para la Evaluación de la Educación Superior, A. C. (CIEES) y Acreditado por el Consejo de Acreditación de la Enseñanza en la Contaduría y Administración, A. C. (CACECA), los empresarios manifiestan que requieren reforzar sus conocimientos en el área fiscal, en el manejo de software y en auditoría. Estos resultados son contrarios a los estudios de Antognolli (2008) quien señala que el Licenciado en Contaduría está preparado especialmente en materia impositiva y en tareas de auditoría.

No obstante que los Licenciados en Contaduría cuentan con la preparación teórica como una de sus fortalezas, los empresarios consideran que les falta capacidad para administrar y el manejo de ciertos programas de cómputo, pues no es lo mismo tener los conocimientos que ponerlos en práctica. Esto es consistente con Hernández (2009) quien indica que los Licenciados en Contaduría requieren contar con un adecuado manejo administrativo y operar eficazmente programas informáticos para hacer más efectiva su labor profesional.

Asimismo, los empresarios indican que los Licenciados en Contaduría necesitan reforzar ciertos valores tales como la responsabilidad, la honestidad y la lealtad, por citar algunos de ellos, lo que es congruente con la literatura de investigación ya que la Organización Mundial del Comercio (OMC) está exigiendo la aplicación de normas de conducta que aseguren el cumplimiento de los estándares mundiales de calidad de estos servicios.

El Licenciado en Contaduría tiene la oportunidad de adquirir mayor experiencia, actualizarse en nuevas teorías y sistemas de cómputo, reforzar sus conocimientos y valores que presentan debilidades con la finalidad de hacer frente a las amenazas actuales de otras Instituciones de Educación Superior tanto públicas como privadas, el avance de las nuevas tecnologías de información, la corrupción y el desempleo. Superar estas deficiencias permitirá contar con un profesional competitivo en el mercado laboral actual, lo que es consistente con Ramos (2010) quien establece que el Licenciado en Contaduría tiene que prepararse y desarrollarse en los nuevos ambientes cada vez más competitivos para hacer frente a los retos que les depara el futuro.

Por otra parte, las Pymes sugieren a la universidad para mejorar la formación de los Licenciados en Contaduría que revise y actualice constantemente sus planes y programas de estudio, pues mucho de lo que se enseña ya está obsoleto y no tiene aplicación real. Para que los Licenciados en Contaduría puedan desarrollar sus competencias es indispensable que dentro de su preparación profesional se incremente la vinculación y práctica con las Pymes. Esto es consistente con Ibarrondo (2011) quien al establecer un puente de enlace entre la docencia y la práctica empresarial a través de visitas guiadas a diversas empresas encontró en los estudiantes 
participantes un incremento en el desarrollo de sus habilidades personales e interpersonales, así como también mayor interés por la adquisición de conocimientos.

El personal docente también requiere estar a la vanguardia en conocimientos, por lo que es necesario su capacitación y actualización constante, lo que es coherente con De Agüero (2001) quien manifiesta que las instituciones de educación superior enfrentan retos que las han obligado a reflexionar sobre la necesidad de hacer cambios en los procesos de enseñanza aprendizaje para adaptarlos a las nuevas condiciones del mercado.

La investigación contribuye al estudio de la formación de Licenciados en Contaduría, un área en la que existe limitada investigación en el contexto mexicano. Los resultados son de utilidad para la institución formadora, en tanto que permitió detectar áreas en las que es necesario fortalecer la formación de estos profesionales. El uso de la metodología cualitativa contribuyó también a obtener una mayor comprensión de la perspectiva de los empleadores y es consistente con el contexto sociocultural en el que se realizó la investigación.

Este estudio se realizó en el año de 2012 y sólo incluyó a los Licenciados en Contaduría de una universidad ubicada en México. Debido a lo anterior, futuras investigaciones deberán incluir una muestra representativa de egresados, así como hacer una revisión de diversos planes de estudio de los centros formadores de los Licenciados en Contaduría. También podría investigarse si los planes y programas de estudio coinciden con los requerimientos de las Pymes, sus necesidades de reestructuración y modernización entre otros; en beneficio de la formación del Licenciado en Contaduría, de las Pymes, del desarrollo económico de su Estado y México.

\section{BIBLIOGRAFÍA}

Allende, C., y MORONES, G. [2006]: Glosario de términos vinculados con la cooperación académica. México: ANUIES. Recuperado el 20 de junio de 2012 de: http://www.anuies.mx/media/docs/convocatorias/pdf/glosariocoopnal2jul06.pdf

Antognolu, S. [2008]: El Contador Público en las PYME. Gestiopolis.com. Recuperado el 8 de marzo de 2012 de: http://www.gestiopolis.com/innovacionemprendimiento/contador-publico-en-las-pymes.htm

Bernabeu, A. [2009]: Competencias requeridas al Contador Público Nacional en el mercado laboral actual. UAD. Facultad de Ciencias Económicas y Jurídicas. Instituto de Investigaciones de la FCEJ (IDICEJ). Recuperado el 17 de junio de 2013 http://www.facpce.org.ar/web201 1/files/img_prof_art_tec/bernabeu.pdf

BIEDMA, E., GÓMEZ, N., Y RUIZ, E. [2011]: El practicum como herramienta de evaluación de las competencias profesionales de los alumnos del Máster de Contabilidad y Auditoría. Educade. Revista de Educación en Contabilidad, Finanzas y Administración de Empresas. No. 2. 113-143. Asociación Española de Contabilidad y Administración de Empresas (aeca). Universidad de Sevilla. Recuperado el 22 de marzo de 2013 de: http://www.educade.es/docs/02/06biedma.pdf

DE AGÜERO, M. [2001]: La formación de administradores y contadores en un nuevo contexto, segunda parte: los nuevos requerimientos. Revista Contaduría y Administración. No. 202. México, Universidad Nacional Autónoma de México. Recuperado el 15 de noviembre de 2012, de: http://www.ejournal.unam.mx/rca/202/RCA20203.pdf

FERNÁNDEZ, J. A. [2001]: "Elementos que consolidan al concepto de profesión. Notas para su reflexión", en Revista Electrónica de Investigación Educativa. Vol. 3, núm. 1. Ensenada Baja California: Universidad Autónoma de Baja California, Instituto de Investigación y Desarrollo Educativo. Recuperado el 5 de octubre de 2012, de: http://www.redalyc.org/articulo.oa?id=15503202 
GHERSI, E., Y AGUIRRE, M. [2010]: Formación y evaluación por competencias del egresado Contador Público de UCES. Buenos Aires: Universidad de Ciencias Empresariales y Sociales (UCES). Recuperado el 17 de junio de 2013 de: http://dspace.uces.edu.ar:8180/xmlui/bitstream/handle/123456789/929/In_fina I_Formaci\%C3\%B3n\%20y\%20evaluacion.pdf? sequence=1

HeRnÁNDEZ, D. [2009]: Perú. "La gestión empresarial y el rol innovador del contador público". Tesis de doctorado. Lima Perú. Recuperado el 7 de octubre de 2012, de: $\quad$ http://www.gestiopolis.com/finanzas-contaduria/contador-publicogestion-mpresarial-ayacucho-peru.pdf

IBARRONDO, P. [2011]: Práctica empresarial y desarrollo de habilidades en el aprendizaje de la Contabilidad de Gestión. Educade. Revista de Educación en Contabilidad, Finanzas y Administración de Empresas. No. 2. 35-50. Asociación Española de Contabilidad y Administración de Empresas (aeca). Universidad de Sevilla. Recuperado el 22 de marzo de 2013 de: http://www.educade.es/docs/02/02-ibarrondo.pdf

INTERNATIONAL FEDERATION OF ACCOUNTANTS (IFAC) [1998]: Guía internacional de educación No. 9: antecedentes académicos, evaluación de la capacidad y de la experiencia de profesionales, requisitos de los contadores profesionales. Contaduría. No. 32, pp. 15-35.

LOPERA, E., GRANDA M., CASTAÑO C., y ROdRíGUez A. [2009]: Hacia un nuevo planteamiento de la contaduría pública frente a la realidad de las micro, pequeñas y medianas empresas (MIPYMES). Adversia. Revista virtual de estudiantes de Contaduría Pública. No. 4 Medellín. Universidad de Antioquía. Recuperado el 8 de marzo de 2012 de: http://aprendeenlinea.udea.edu.co/ revistas/index.php/adversia/article/viewFile/2031/1680

MONTERO, H. Y ZAÁ, R. [2009]: Ética, normatividad y calidad. XXVIII Conferencia Interamericana de Contabilidad. Cancún 2009. Recuperado el 23 de marzo de 2012

de: http://www.colegiodecontadorescochabamba.org.bo/descargas/ octubre09total.pdf

ORganizaCión PARA LA COOPERACIÓN Y EL DESARROLLO ECONÓMICO [2005]: La definición y selección de competencias clave: Resumen ejecutivo. Recuperado el 20 de septiembre de 2012 de: http://www.deseco.admin.ch/bfs/deseco/en/index/03/02.parsys.78532.downl oadList.94248.DowloadFile.tmp/2005.dscexecutivesummary.sp.pdf

Quirós, E. H. (2006]: Educación Contable. Competencias formativas y mercado profesional contable. Revista Internacional Legis de Contabilidad \& Auditoría. 143-195. Recuperado el 18 de junio de 2013 de: http://scienti.colciencias.gov.co:8084/publindex/docs/articulos/16922913/33/144.pdf

RAmOS, J. [2010]: El contador público en la actualidad. Buenas tareas. Recuperado el 23 de marzo de 2012 de: http://www.buenastareas.com/ensayos/ElContador-Publico-En-La-Actualidad/376230.html

StAKE, R. E. [1994]: "Case studies". pp 236-247 in Norman K. Denzin (1994). Handbook of Qualitative Inquiry. Thousand Oaks: CA.

Tobón, S., PImienta, J., y García, J. [2010): Secuencias didácticas: aprendizaje y evaluación de competencias. México: Pearson.

UNIVERSIDAD AUtÓNOMA DE CAMPECHE [2011]: Facultad de Contaduría y Administración. Recuperado el 3 de agosto de 2012 de: http://ftp.vacam.mx/ofertaeducativa/facultad/seccion/329/facultad-de-contaduria-yadministracion/antecedentes-historicos

UNIVERSIDAD AUTÓNOMA DE CAMPECHE [2012): Licenciatura en Contaduría. Recuperado el 7 de agosto de 2012 de: http://fca.vacam.mx/?modulo_micrositio=paginas\&acciones_micrositio=ver\&i d_pagina $=f w==$ 
VAlerA, L. [2009): Propuesta de competencias profesionales para los estudiantes de la carrera de Contabilidad y Finanzas. Cuadernos de Educación y Desarrollo. Revista Académica Semestral. Vol. 1 No. 1. Recuperado el 18 de junio de 2013 de: http://www.eumed.net/rev/ced/01/lvf.htm 